International Journal of Engineering \& Technology, $7(4.5)(2018) 309-312$
International Journal of Engineering \& Technology
WPC
Website: www.sciencepubco.com/index.php/IJET
Research paper

\title{
Total Harmonic Modulation Distortion of an Injection-Locked Fabry-Perot Laser Diode Directly Modulated by a Multi-harmonic RF Signal
}

\author{
Meenakshi Chakraborty ${ }^{1}$, Taraprasad Chattopadhyay ${ }^{2 *}$ \\ ${ }^{1}$ The Department of Physics, Visva-Bharati (Central University), Santiniketan 731235, West Bengal, India. \\ ${ }^{2}$ Department of Physics, Visva-Bharati (Central University), Santiniketan 731235, West Bengal, India. \\ *Corresponding author E-mail:tara_vb@hotmail.com
}

\begin{abstract}
This paper describes effects of low-level light injection into a directly modulated Fabry-Perot laser diode on the external total harmonic modulation distortion when the external fundamental modulating signal is multi-harmonic contaminated. Necessary theory of the injected FPLD has been developed and an expression for total harmonic distortion of the modulating signal has been derived considering lowlevel light injection. The total harmonic modulation distortion has been experimentally measured for the RF modulation source and for the injection-locked slave FPLD output after photodetection.
\end{abstract}

Keywords: Direct Modulation; Injection Locking; Lockband; Semiconductor Laser;Total Harmonic Distortion.

\section{Introduction}

Semiconductor laser diodes are playing major roles in wideband electromagnetic communication which can perform many functions such as coherent sources, amplifiers of optical FM and PM signals and modulation converters in the injection-locked mode of operation. Demodulation of optical PM/FM signals can also be done in the latter mode. Recently ultradense wavelength division multiplxed (UDWDM) passive optical network (PON) [1-2] is being implemented where multiple coherent sources are necessary for the transmission of many channels simultaneously. The basic scheme of modulation and detection in optical communication is the intensity modulation (IM) and direct detection (DD) in a photodiode. Some workers have studied theoretically the phenomenon of nonlinear distortion reduction [3-5] in a laser diode (LD) by high power lightwave injection into the modulated LD. This distortion is generated internally in the LD itself due to its nonlinearity in the light output vs. bias current characteristics.

In this paper, we investigate another phenomenon of the effect of low-level light injection on a LD directly modulated by an external multi-harmonic distortion-contaminated RF signal. The primary difference between the existing reports on distortion reduction and the present study is that, in the earlier studies the distortion is internal, whereas in the present study it is being added externally with the fundamental modulating signal. The transformation of second harmonic IM distortion in an IM-DD fiber optic link has been studied by the author [6]. Depending upon the availability of the RF modulation source, we have measured the total harmonic distortion (THD) of the RF source itself and of the injectionlocked Fabry-Perot (FP) slave laser diode (SLD) directly modulated by a distorted modulating signal under low-level optical injection [7-13]. The RF source operates in the range of $100 \mathrm{MHz}-400$ MHz.
The external harmonic distortion may arise from the nonlinearity of the transducer producing sonic to electrical signal conversion in the case of direct voice signal baseband modulation, or it can originate from the nonlinearity of the RF source. The RF signal can also act as a subcarrier of the baseband signal.

\section{Analysis}

The experimental setup is shown in Fig. 1

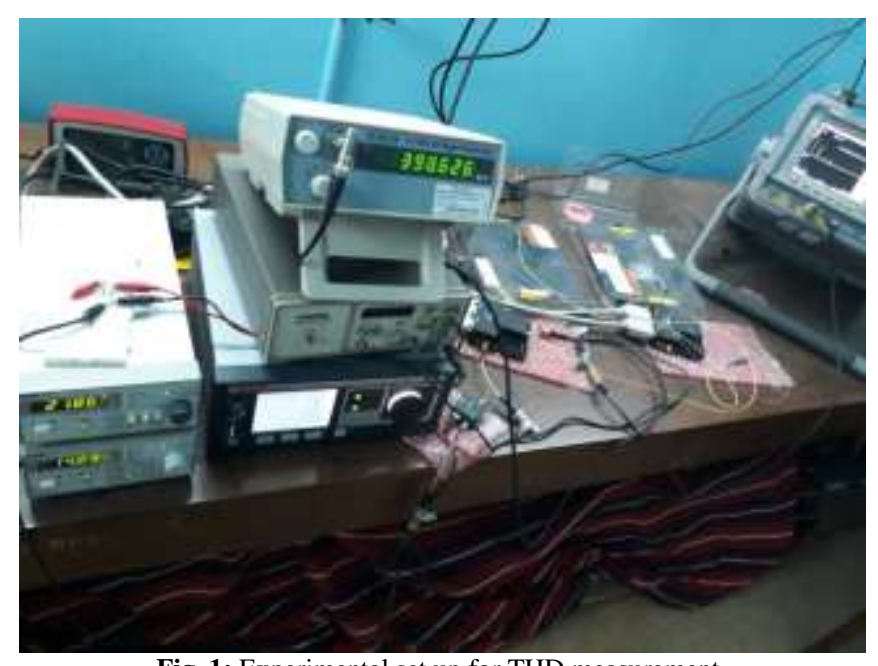

Fig. 1: Experimental set up for THD measurement

Let the RF modulation signal be represented as,

$v_{m}(t)=\sum_{n=1}^{N} V_{n} \sin \left(n \omega_{m} t+\theta_{n}\right)$ 
where $V_{n}$ is the voltage modulation amplitude of the $n$-th harmonic. $n=1$ corresponds to the fundamental modulation and $n=2$, $3, \ldots . \mathrm{N}$ correspond to second harmonic and higher order harmonics respectively. These harmonics are undesired components of the modulation signal and are small in amplitude compared with the fundamental. These undesired harmonics are generated due to nonlinearity of the device or of the transducer which produce the modulating signal. The intensity modulation of the SLD is described by the equation:

$$
I(t)=I_{0}\left[1+\sum_{n=1}^{N} m_{I n} \sin \left(n \omega_{m} t+\theta_{n}\right)\right]
$$

where $\mathrm{I}_{0}$ is the average light intensity of slave laser and $\mathrm{m}_{\mathrm{In}}$ the intensity modulation (IM) index at $n$-th harmonic component of modulation. The corresponding power modulation is given by,

$$
P(t)=P_{m}\left[1+\sum_{n=1}^{N} m_{I n} \sin \left(n \omega_{m} t+\theta_{n}\right)\right]
$$

where, $\mathrm{P}_{\mathrm{m}}$ is the average light output power of the modulated slave laser diode (SLD). The frequency chirp due to direct modulation is described by Koch-Bower's relation as,

$$
\Delta v(t)=-\frac{\alpha}{4 \pi} \frac{\partial}{\partial t} \ln P(t)
$$

where $\alpha$ is the linewidth enhancement factor of the slave FPLD The phase modulation generated by this chirp is given by,

$$
\begin{aligned}
\psi(t) & =2 \pi \int \Delta v(t) d t \\
& =-\frac{\alpha}{2} \ln P(t) \\
& =\psi_{0}+\sum_{n=1}^{N} m_{f n} \sin \left(n \omega_{m} t+\theta_{n}\right)
\end{aligned}
$$

where $\psi_{0}=-\frac{\alpha}{2} \ln P_{m}$. The FM Index, $m_{f n}=\frac{\alpha}{2} m_{I n}$. Here we have assumed $m_{I n}<<1$ for $\mathrm{n}=1,2, \ldots \mathrm{N}$, so that

$$
\ln \left[1+\sum_{n=1}^{N} m_{I n} \sin \left(n \omega_{m} t+\theta_{n}\right] \approx-\sum_{n=1}^{N} m_{I n} \sin \left(n \omega_{m} t+\theta_{n}\right)\right]
$$

The electric field of the lightwave injected into the bias current modulated SLD can be expressed as,

$$
E_{i n}(t)=\sqrt{P_{i n}} e^{j\left(\omega_{c} t+\theta_{i}\right)}
$$

where $\mathrm{P}_{\text {in }}$ is the injection power, $\omega_{\mathrm{c}}$ is the radian frequency and $\theta_{i}$ is an arbitrary phase angle of the injection lightwave.

Electric field of free running slave laser in absence of light injection is :

$$
E_{f s}(t)=E_{o f} e^{j\left(\omega_{0} t+\psi(t)\right)}
$$

where, $\omega_{0}$ is the radian frequency of free running slave laser diode and $\psi(\mathrm{t})$ an arbitrary phase angle.

$E_{o f}^{2}(t)=P_{m}\left[1+\sum_{n=1}^{N} m_{I n} \sin \left(n \omega_{m} t+\theta_{n}\right)\right]$

The electric field carrying n-th harmonic modulation in the output lightwave from the locked SLD can be written as,

$$
E_{o n}(t)=E_{L n}(t) e^{j\left(\omega_{c} t+\phi_{0 n}(t)\right)}
$$

where $E_{L n}(t)$ is the amplitude modulated electric field while $\phi_{0 n}(t)$ represents output phase modulation. Under locked condition, the frequency of the SLD becomes equal to that of the injection lightwave. $E_{\mathrm{Ln}}(\mathrm{t})$ is of the form,

$E_{L n}(t)=\sqrt{P_{m}}\left[1+\sum_{n=1}^{N} m_{0 n} \sin \left(n \omega_{m} t+\theta_{n}\right)\right]^{1 / 2}$

where $m_{0 n}$ (for $n=1,2, \ldots . N$ ) is the output IM index of the $n$-th harmonic modulating signal. $\theta_{\mathrm{n}}($ for $\mathrm{n}=1,2, \ldots \mathrm{N}$ ) is an arbitrary phase angle of the $\mathrm{n}$-th harmonic which is not expected to change due to light injection.

The amplitude equation of the injected slave LD carrying $\mathrm{n}$-th harmonic modulation is written as,

$$
\begin{aligned}
& \frac{2 Q}{\omega_{0}} \frac{1}{\left|E_{L n}(t)\right|} \frac{d\left|E_{L n}(t)\right|}{d t}=-2+\left[C_{1}-C_{2} \frac{\left|E_{L n}(t)\right|^{2}}{\left|E_{0 f}(t)\right|^{2}}\right] \\
& \times\left[\frac{\left|E_{i n}(t)\right|}{\left|E_{L n(t)}\right|} \cos \left(\theta_{i}-\phi_{0 n}(t)\right)+1\right]
\end{aligned}
$$

where $C_{1}=\left(\mu-\frac{1}{\mu}\right) l \alpha_{m}$

$C_{2}=\left(\mu-\frac{1}{\mu}\right) \lg _{0} \frac{\left|E_{o f}\right|^{2}}{\left|E_{s}\right|^{2}}$

$Q=\left(\mu-\frac{1}{\mu}\right) \frac{2 \pi l}{\lambda}$

where ' $l$ ' is the LD cavity length, $\mu$ is the refractive index of the active medium of the LD, $\alpha_{m}$ is the mirror loss, $g_{0}$ is the linear gain of the LD, Q is the external Q-factor of the FPLD, $E_{0 f}$ is the electric field amplitude of the SLD and $E_{s}$ is the saturation electric field of the SLD. $\lambda$ is the operating wavelength of the slave FPLD. We assume output phase modulation in the form,

$$
\phi_{0 n}(t)=\phi_{a v}+\sum_{n=1}^{N} \phi_{n} \sin \left(n \omega_{m} t+\theta_{n}\right)
$$

where, $\phi_{n}$ is the output phase modulation amplitude of the n-th harmonic. Let $\xi=\theta_{i}-\phi_{a v}$ be the average phase error of the locked SLD. Substituting (6), (8), (10) and (15) in (11) and applying the Principle of Harmonic balance we get, 


$$
\frac{m_{0 n}^{2}}{m_{I n}^{2}}=\frac{\left(x_{3} \frac{\phi_{n}}{m_{I n}}+x_{4}\right)^{2}}{n^{2} x_{1}^{2}+x_{2}^{2}}
$$

where $x_{1}=\frac{\omega_{m}}{\omega_{0}} Q$

$x_{2}=C_{2}+\frac{1}{2}\left(C_{1}+C_{2}\right) \sqrt{\frac{P_{i n}}{P_{m}}} \cos \xi$

$x_{3}=\left(C_{1}-C_{2}\right) \sqrt{\frac{P_{i n}}{P_{m}}} \sin \xi$

$x_{4}=C_{2}\left[1+\sqrt{\frac{P_{i n}}{P_{m}}} \cos \xi\right]$

Here, we have assumed $J_{0}\left(\phi_{n}\right) \approx 1$ and $J_{1}\left(\phi_{n}\right) \approx \frac{\phi_{n}}{2}$, since $\phi_{n}$ are much less than unity. We have also neglected products of the form $\phi_{i} \phi_{j}(\mathrm{i}, \mathrm{j}=1,2, \ldots \mathrm{N})$ and higher order products due to smallness.

The output phase modulation amplitude can be estimated from the phase equation given below:

$$
\begin{aligned}
& \frac{2 Q}{\omega_{0}} \frac{d \phi_{0 n}(t)}{d t}=\left[C_{1}-C_{2} \frac{\left|E_{L n}(t)\right|^{2}}{\left|E_{0 f}(t)\right|^{2}}\right] \\
& \times\left[\frac{\left|E_{i n}(t)\right|}{\left|E_{L n}(t)\right|} \sin \left(\theta_{i}-\phi_{0 n}(t)\right)\right]-2 \delta Q
\end{aligned}
$$

where $\delta=\frac{\omega_{c}-\omega_{0}}{\omega_{0}}$ is the normalized detuning of the SLD from the injection $\mathrm{CW}$ lightwave. Here, we have neglected the effect of linewidth enhancement factor, $\alpha$, in deriving the phase equation.

Substituting $E_{L n}(t)$ and $\phi_{O n}(t)$ in (21) and applying the Principle of harmonic balance we get,

$$
\begin{aligned}
& \frac{\phi_{n}^{2}}{m_{I n}^{2}}=\frac{\left(y_{3}-y_{4}\right)^{2}}{n^{2} y_{1}^{2}+y_{2}^{2}} \\
& \text { where } y_{1}=\frac{2 Q \omega_{m}}{\omega_{0}} \\
& y_{2}=\left(C_{1}-C_{2}\right) \sqrt{\frac{P_{i n}}{P_{m}}} \cos \xi \\
& y_{3}=\frac{1}{2}\left(C_{1}+C_{2}\right) \sqrt{\frac{P_{i n}}{P_{m}}} \sin \xi
\end{aligned}
$$

$y_{4}=C_{2} \sqrt{\frac{P_{i n}}{P_{m}}} \sin \xi$

The dc phase equation which is obtained from (21) by equating $\mathrm{dc}$ terms from both sides can be written as,

$\left(C_{1}-C_{2}\right) \sqrt{\frac{P_{i n}}{P_{m}}} \sin \xi-2 \delta Q=0$

$\omega_{c}-\omega_{0}=\left(C_{1}-C_{2}\right) \sqrt{\frac{P_{i n}}{P_{m}}} \frac{\omega_{0}}{2 Q} \sin \xi$

At the extremities of the lockband, $\xi= \pm \pi / 2$.

$\Delta \omega_{U}=\frac{\omega_{0}}{2 Q}\left(C_{1}-C_{2}\right) \sqrt{\frac{P_{i n}}{P_{m}}}$

$=\Delta \omega_{L}$

where $\Delta \omega_{U}$ is the upper side lockband corresponding to the case $\xi=+\pi / 2$ and $\Delta \omega_{L}$ is the lower side lockband corresponding to the case $\xi=-\pi / 2$. The lockband is symmetrically located around the centre frequency, $\omega_{0}$, which is the free-running frequency of the SLD. This is due to the fact that we have neglected the effect of linewidth enhancement factor $(\alpha)$ in the phase equation (21) which would otherwise give rise to asymmetry in locking.

The output optical power of the locked SLD is detected in a $1.2 \mathrm{GHz}$ bandwidth InGaAs photodiode (PD). The output current of the PD is proportional to $\mathrm{m}_{0 \mathrm{n}}$ for the $\mathrm{n}$-th harmonic modulation. So, the $\mathrm{n}$-th harmonic modulation power in the electrical domain is proportional to $m_{0 n}^{2}$ (for $\left.\mathrm{n}=1,2, \ldots \mathrm{N}\right)$. The total harmonic distortion (THD) of the fundamental modulation is defined as :

$\mathrm{D}=($ Total harmonic electrical power $) /($ Total electrical power of the detected signal).

The THD can be mathematically expressed as :

$D=\frac{\sum_{n=2}^{N} m_{0 n}^{2}}{\sum_{n=1}^{N} m_{0 n}^{2}}$

\begin{tabular}{|c|c|c|}
\hline $\begin{array}{c}\text { Modulation Fre- } \\
\text { quency }\left(\mathrm{f}_{\mathrm{m}}\right) \text { in } \mathrm{MHz}\end{array}$ & $\begin{array}{c}\text { THD (in \%) at the } \\
\text { Input }\end{array}$ & $\begin{array}{l}\text { THD (in \%) at the } \\
\text { Output of the } \\
\text { ILSLD }\end{array}$ \\
\hline 100 & 2.68 & 1.91 \\
\hline 150 & 4.72 & 1.71 \\
\hline 200 & 6.47 & 7.02 \\
\hline 350 & 6.89 & 4.14 \\
\hline 400 & 3.89 & 13.57 \\
\hline
\end{tabular}

This THD has been measured experimentally by using a $9 \mathrm{KHz}-3$ $\mathrm{GHz}$ Agilent microwave spectrum analyzer (model : E4403B). The measured data are shown in Table 1.

Table 1: Experimentally measured values of THD at the input and at the output of injection locked SLD (ILSLD) 


\section{Conclusion}

In this paper, we have measured experimentally the total harmonic modulation distortion of an injection-locked Fabry-Perot laser diode when the input modulating RF signal contains fundamental as well as its harmonics. We have also developed a theory of the injection-locked Fabry-Perot laser diode which can explain the observed THD. It has been experimentally observed that optical injection reduces the amplitude of the fundamental modulating signal. Depending upon the levels of multiharmonics at the modulation input, the THD, which is defined as the ratio of the total harmonic power to the total electrical signal power of modulation, of the injected laser can be reduced or enhanced. This is a novel observation which is being reported for the first time in literature.

\section{References}

[1] M. Chakraborty and T. Chattopadhyay, "A novel scheme for UD WDM-PON broadband access network using injection-locked phase-to-intensity modulation converter", Journal of optical Communications, in press, (2017), DOI : 10.1515/joc-2017-0085.

[2] M. Chakraborty and T. Chattopadhyay, "A scheme for UDWDMPON broadband access network using a mode locked laser diode and optical injection locking", Journal of Optical Communications, in press, 2017, DOI : 10.1514/joc-2017-0019.

[3] M. Chakraborty and T. Chattopadhyay, "Optical modulation enhancement through $\mathrm{CW}$ injection locking of a sinusoidally modulated Fabry-Perot laser diode" Journal of Optical Communications, 2017, DOI: 10.1515/joc-2016-0157.

[4] T. Chattopadhyay, P. Bhattacharyya, C. Ghosh, "Conservation of AM index of a RF subcarrier in IM-DD optical link," OptikInternational Journal for Light and Electron Optics, Vol. 127, No.13, (2016) 5448-5451.

[5] T. Chattopadhyay, P. Bhattacharyya and C.Ghosh, "Linewidth enhancement factor measurement of a Fabry-Perot laser diode through narrowband optical FM generation", Journal of Optical Communication, Nov. 2015, DOI: 10.1515/joc-2015-0022 (in press).

[6] C. Ghosh and T. Chattopadhyay, "Measurement of a secondharmonic distortion of the detected signal in an IM-DD fiber optic link when the input IM is second harmonic contaminated, "Photonics Letters of Poland", Vol. 7, No. 3, (2015), pp. 84-86.

[7] T. Chattopadhyay and P. Bhattacharyya, "Linewidth enhancemen factor and amplification of angle-modulated optical signals in injection-locked quantum cascade lasers", Journal of Optical Communications, Vol. 37, Issue 1, (2016), pp. 23-30, DOI: 10.1515/ joc2015-0009.

[8] C. Ghosh and T. Chattopadhyay, "Efficient FM-IM Conversion in an injection-locked Fabry-Perot laser diode, "International Journa of Electronics and Communication Engineering \& Technology (IJECET)", Vol. 7, No. 2, (2016), pp. 33-40.

[9] M. Bhattacharya, B. Sarkar and T. Chattopadhyay, "Optical generation of millimeter and sub millimeter waves through optical sideband injection locking of semiconductor lasers", IEEE Photonics Technology Letters, vol. 14, no. 11, (2002), pp.1611-1613.

[10] M. Bhattacharya and T. Chattopadhyay, "A method for generation of optical FM signal through injection locking", J. Lightwave Technology, Vol. 16, (1998), pp.656-660.

[11] T. Chattopadhyay and M. Bhattacharya, "Sub-millimeter wave generation through optical four wave mixing using injection locked semiconductor laser", J. Lightwave Technology, Vol. 20, (2000), pp.502-506.

[12] M. Bhattacharya and T. Chattopadhyay, "Influence of adjacent channel interference on frequency-modulated WDM optical communication system", J. Lightwave Technology, Vol. 17, (1999), pp.2516-2519.

[13] S. Kobayashi and T. Kimura, "Injection locking in AlGaAs semiconductor laser", IEEE J. Quantum Electronics, Vol. QE-17, No. 5, (1981), pp.681-689. 